\title{
Effects of Impact Speeds, Fall Postures and Cortical Thicknesses on Femur Fracture by Incremental Element Deletion Based Finite Element Analysis
}

\author{
Yangyang Cui ${ }^{1}$, Dingding Xiang ${ }^{1}$, Liming Shu ${ }^{1}$, Zhenhua Liao ${ }^{1}$, Zhilin Zhang $^{1}$, and \\ Weiqiang $\mathrm{Liu}^{1}$ \\ ${ }^{1}$ Affiliation not available
}

March 5, 2021

\begin{abstract}
Numerical simulation of the proximal femur could provide an effective approach to predict the femoral fracture risk. However, most of the extant numerical simulations are static simulations, which cannot accurately reflect the dynamic growth process of cracks. In this study, the dynamic simulation by incremental element deletion (IED) based finite element analysis (FEA) was developed on femur fracture analysis and compared with two widly-used approaches (XFEM and USDFLD). It was also evaluated using the in-vitro loading test. Moreover, the effects of different impact speeds, fall postures, and cortical thicknesses on fracture types and mechanical responses were investigated. Impact speeds cause different crack propagation behaviors of the femoral neck. Falling forward was less likely to cause proximal femoral fracture compared with falling backward. The model with thin cortical bone was prone to fracture. These provides a theoretical basis and method for predicting femoral fracture risk in the elderly.
\end{abstract}

Effects of Impact Speeds, Fall Postures and Cortical Thicknesses on Femur Fracture by Incremental Element Deletion Based Finite Element Analysis

Yangyang Cuia,b, Dingding Xiang, ${ }^{\mathrm{b}, \mathrm{c},}{ }^{*}$, Liming Shu ${ }^{\mathrm{d}}$, Zhenhua Liao ${ }^{\mathrm{c}}$, Zhilin Zhang ${ }^{\mathrm{e}}$, Weiqiang Liu $\mathrm{L}^{\mathrm{b}, \mathrm{c}, \mathrm{e}^{*}}$

${ }^{a}$ Division of Advanced Manufacturing, Tsinghua Shenzhen International Graduate School, Tsinghua University, Shenzhen, 518055, China

b State Key Laboratory of Tribology, Department of Mechanical Engineering, Tsinghua University, Beijing, 100084, China

${ }^{\mathrm{c}}$ Key Laboratory of Biomedical Materials and Implant Devices, Research Institute of Tsinghua University in Shenzhen, Shenzhen, 518057, China

d Department of Mechanical Engineering, School of Engineering, The University of Tokyo, 1138656, Japan

${ }^{e}$ Department of Biomedical Engineering, Tsinghua University,Beijing 100084, China

\section{${ }^{*}$ Corresponding author.}

E-mail address: weiqliu@hotmail.com (W.Q. Liu) and Totoptop@foxmail.com (D.D. Xiang)

\section{Abstract}


Numerical simulation of the proximal femur could provide an effective approach to predict the femoral fracture risk. However, most of the extant numerical simulations are static simulations, which cannot accurately reflect the dynamic growth process of cracks. In this study, the dynamic simulation by incremental element deletion (IED) based finite element analysis (FEA) was developed on femur fracture analysis and compared with two widly-used approaches (XFEM and USDFLD). It was also evaluated using the in-vitro loading test. Moreover, the effects of different impact speeds, fall postures, and cortical thicknesses on fracture types and mechanical responses were investigated. Impact speeds cause different crack propagation behaviors of the femoral neck. Falling forward was less likely to cause proximal femoral fracture compared with falling backward. The model with thin cortical bone was prone to fracture. These provides a theoretical basis and method for predicting femoral fracture risk in the elderly.

Keywords: Fracture; Femur; Finite element analysis; Numerical simulation; Bone

\section{1 | Introduction}

The number of patients with osteoporotic fractures continues to grow every year, and it is expected that the number of patients with osteoporotic fractures around the world will be 1.55 billion by $2050 .{ }^{1}$ Among them, the survival rate of proximal femoral fracture is the lowest. There is currently one proximal femoral fracture every $3 \mathrm{~s}$ in the world. ${ }^{2}$ The disability rate of proximal femoral fracture is more than $50 \%$, and the mortality rate can reach $8.4 \% 36 \%$ within one year. ${ }^{3}$ Even surviving patients are often accompanied by various complications that seriously affect the quality of life. ${ }^{4}$ It is indicated that about $90 \%$ of proximal femoral fractures are caused by falls, the direction and speed of the fall are the main factors that affect the femoral fracture. ${ }^{5}$ However, not all falls can cause proximal femoral fractures. Compared with the elderly, falls, which occur on young and middle-aged people in the same way and with the same external factors, rarely cause similar fractures. This may be related to the thickness of cortical bone with age. ${ }^{6}$ Nakamura et al. ${ }^{7}$ also pointed out that the proximal femoral fractures are related to different fall conditions (fall postures, impact speeds) and the thicknesses of femoral cortical bones from a biomechanical perspective. If the femoral fractures can be predicted at an early stage, fractures will be easier to treat, which can also reduce the pain of patients. ${ }^{8}$ Therefore, a comprehensive understanding of the proximal femoral fracture factors such as fall postures, impact speeds, and the thicknesses of femoral cortical bones need to be studied.

With the development of computer modeling technology, numerical simulation becomes a useful tool for predicting femoral early fracture behavior. The numerical simulation has the advantages of low cost and reusable model, which has been successfully applied to bone fracture. ${ }^{9} \mathrm{Li}$ et al. ${ }^{10}$ conducted a three-point bending mechanical test on a cortical bone sample with a single-edge incision. Based on the result of the test, an extend finite element method (XFEM) was developed and used to analyze its deformation and cracks. Nicolas et al. ${ }^{11}$ also put forward a finite element method for crack growth without remeshing, it was just XFEM. Giambini et al. ${ }^{12}$ studied the feasibility of the XFEM based on quantitative computed tomography images, and analyzed the feasibility of predicting the risk of vertebral fracture. The results showed that the fracture modes predicted by the XFEM are in good agreement with the experimental observations. However, XFEM can only simulate the initial position of cracks and extremely small cracks due to convergence issues. ${ }^{13,14}$ Besides, Ali et al. ${ }^{15}$ found the predictions of fracture load differed by approximately $50 \%$ due to sensitivity to local material properties. Additionally, the user subroutine to redefine field variables at a material point (USDFLD) is another widely used approach in bone fracture analysis. Giner et al. ${ }^{14}$ used the USDFLD method to establish a numerical model of a single bone block under a compressive load, and simulated the failure of a single bone element under radial compression. The results showed that the interlaminar shear stress was under the compressive load. The main reason for bone unit damage is basically consistent with the experimental results of other authors. ${ }^{16}$ However, the existing numerical simulation methods are mostly static simulations. It is difficult to reflect the dynamic expansion process of cracks. The accuracy of predicting femoral fracture risk in the elderly need to be improved to predict the people who are easy to fracture in order to minimize the consequences.

In this study, the dynamic simulation with incremental element deletion (IED) based finite element analysis (FEA) on femur fracture analysis was developed and compared with XFEM and USDFLD, which was also 
evaluated with the in-vitro loading test. Besides, the method developed was further used to simulate the effects of different impact speeds, fall postures, and cortical thicknesses on fracture types and mechanical responses. It potentially provides a trust worthy method for indicating the femoral fracture risk in the elderly, which can provide reliable theoretical guidance for clinical orthopedics and evaluation of surgical procedures.

\section{2| Materials and Methods}

Five newly designed fourth-generation composite femurs provided by Sawbones (Sawbones, Pacific Research Laboratories, Vashon, USA) were used to investigate fracture behaviors. The composite femur was first CT scanned. Three numerical simulation methods were verified and compared by mechanical tests. The strains of 8 positions on the surfaces of composite femurs were collected in the elastic tests. The fracture loads and crack propagation paths of composite femurs were collected in the fracture tests. Numerical simulation is mainly comprised of three methods: XFEM, user subroutine USDFLD, and the IED-based secondary development.

\subsection{Materials and CT scanning}

The composite femurs comprehensively consider a large number of population data, which are similar and representative to real human bones. ${ }^{17}$ The outer region with variable thickness can simulate cortical bone and an inner region of foam has similar cancellous bone characteristics. CT scanning was performed for the entire composite femur with a slice thickness of $2.5 \mathrm{~mm}$ and a pixel width of $0.938 \mathrm{~mm}$ (GE MEDICAL SYSTEMS/LightSpeed 16 Computed Tomography Scanner System, 80 kV, 443.52 mAs, $512 \times 512$ matrix, 52 images).

\section{2 | Mechanical Tests}

Mechanical tests were performed on the composite proximal femurs. Two different tests were carried out in five specimens: one is the strain measurment in the elastic regime at 8 points of the bone surface, and the other is a fracture test to obtain the fracture load and fracture morphology. Two angles ( $\alpha$ and $\beta$ ) were defined to describe the placement direction of the composite femur in the test and fall posture in the numerical simulation, where the angle $\alpha$ was $0^{\circ}-135^{\circ}$ with reference to the long axis of the femur in the frontal plane, and $\beta$ was $0^{\circ}-45^{\circ}$ with reference to the femoral neck axis in the horizontal plane, as shown in Figure 1a-b. The distal end of the composite proximal femur was fixed with a clamp. $\alpha$ angle of $10^{\circ}$ and $\beta$ angle of $15^{\circ}$ were used to represent the placement direction of human. ${ }^{18,19}$ 


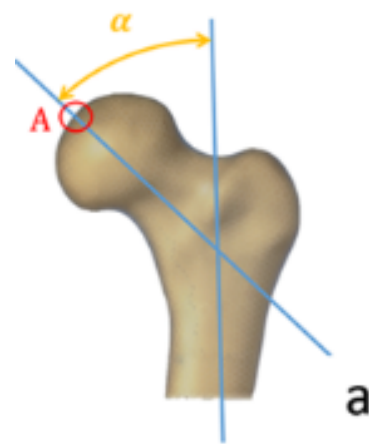

a)

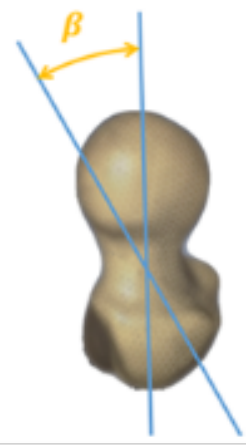

b)

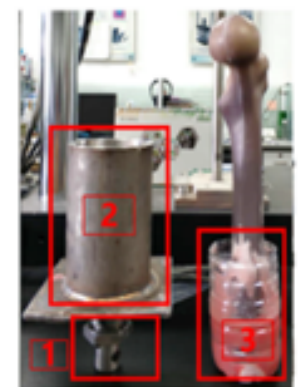

c)
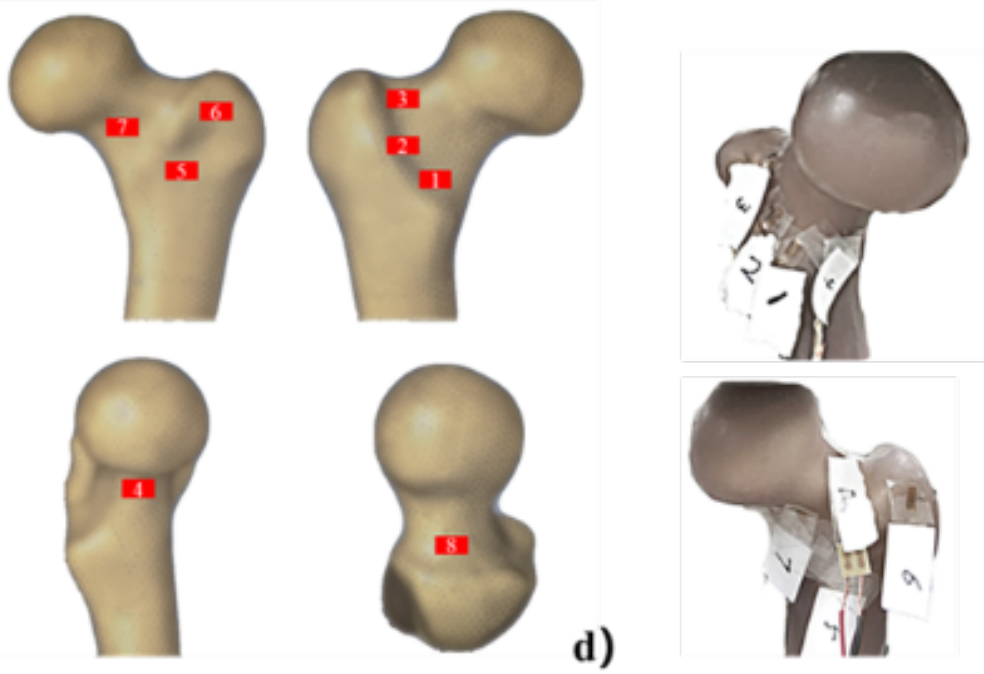

e)

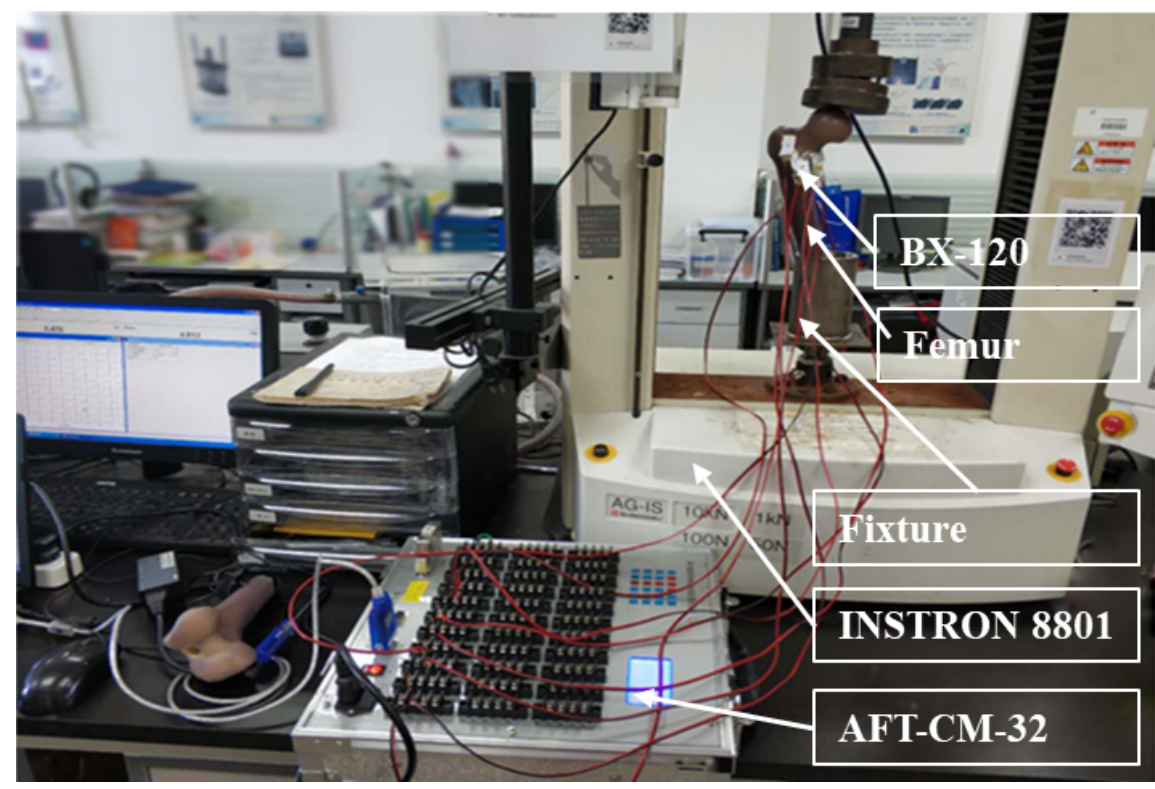


Figure 1 The experimental layout of mechanical testing. (Two angles were defined, $a$ and $\beta$, to describe the placement direction of the composite femur during the test and fall posture in the numerical simulation. Point A was the central position of the spherical region surface with a $35 \mathrm{~mm}$ diameter on the femoral head. a) The angle $a\left(0^{\circ}-135^{\circ}\right)$ with reference to the long axis of the femur in the frontal plane; b) The angle $\beta$ $\left(0^{\circ}-45^{\circ}\right)$ with reference to the femoral neck axis in the horizontal plane; c) Fixation method and device of composite femur. The composite femur was fixed by a disposable fixation container in frame 3 , and then it was put into fixture 2, and finally fixed on the mechanical testing machine by the nut of frame 1; d) The positions of strain measurement during the numerical simulation; e) The positions of strain measurement during mechanical tests; f) experimental device of composite femur compression. The same reference numbers in d) and e) correspond to the same positions.)

The composite femur was tested on a $10 \mathrm{kN}$ universal hydraulic testing machine (INSTRON 8801, load cell $10 \mathrm{kN}$ ). As shown in Figure 1c, the distal end of the composite femur was inserted into the dental powder mixture to maintain the composite femur with $\alpha$ angle of $10^{\circ}$ and $\beta$ angle of $15^{\circ}$, and it was fixed for about 30 min - 60 min. During the test, 8 strain gauges (Qinhuangdao Aft, BX-120) were used to measure the strains at 8 different surface positions. The specific locations where the strain gauges were pasted, as shown in Figure 1d-e. The load was increased to different values $(250 \mathrm{~N}, 500 \mathrm{~N}$ and $750 \mathrm{~N})$ with a speed of 3 $\mathrm{mm} / \mathrm{min}$ to maintain quasi-static condition. Once the elastic regime was analysed for each femur, the load on the femoral head was increased until fracture occurred. The applied force was registered and the fracture load was estimated as the maximum peak of the curve force/displacement followed by a sudden drop of the applied force. Data acquisition was performed immediately when the target loads were reached, which could avoid material relaxation. The strain signals at the 8 points on the surfaces of the composite femurs were sampled at $0.5 \mathrm{~Hz}$ with a 32-channel strain measuring instrument (Qinhuangdao Aft, AFT-CM-32). The whole experimental device of composite femur compression was shown in Figure 1f.

The fracture loads and crack propagation paths of composite femurs were obtained by fracture tests. Because the composite femurs were loaded in the elastic range, no damage occurred. So, the fracture tests could be performed in the same samples. The same fixing method as the previous tests was used, then they were loaded at a speed of $3 \mathrm{~mm} / \mathrm{min}$ until they were broken. From these tests, the fracture loads and crack propagation paths were obtained.

\section{3 | Numerical Simulation}

\subsection{1 | Mesh convergence analysis}

Mimics (Materialise Inc, Leuven, Belgium) was used to construct the finite element model of composite proximal femur. Abaqus (Simulia Inc, Providence, RI, USA) was used to divide the composite proximal femur model into two 3D solid parts. Because of the different materials of composite femurs, they were endowed with two material properties, i.e., cancellous bone and cortical bone. The loading conditions of simulation were exactly the same as that of mechanical tests. Similarly, the strains at the surface positions of the composite femur models were collected when the loading force was $250 \mathrm{~N} / 500 \mathrm{~N} / 750 \mathrm{~N}$. Besides, the fracture loads and the crack propagation paths were collected when the composite femurs were damaged. 

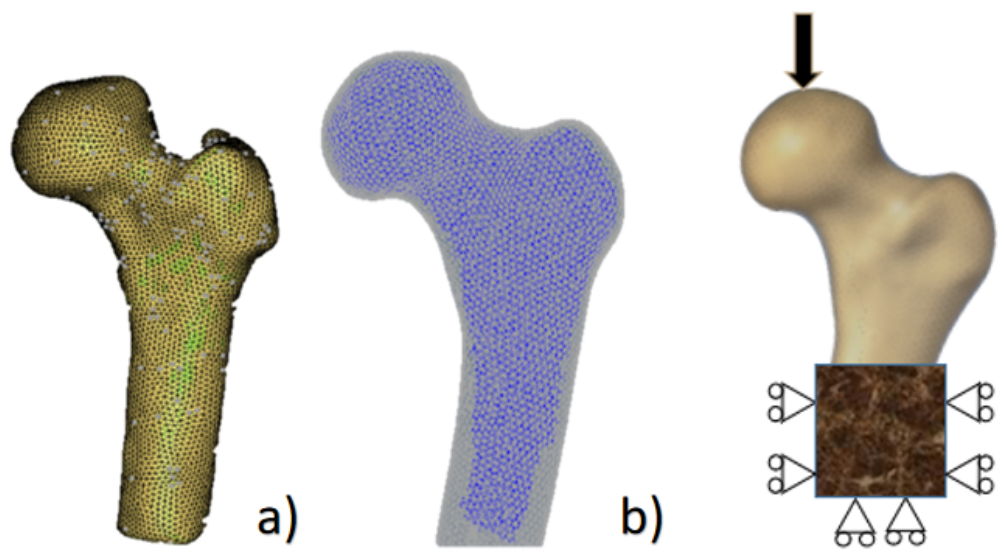

c)

Figure 2 The composite proximal femur with three-dimensional tetrahedral mesh and materials and loading condition. a)The meshed composite proximal .b)The composite proximal femur with two materials, the outer transparent part is cortical bone, and the inner opaque part is cancellous bone. c) the boundary condition and loading condition.

Element size of the finite element model is very important for the accuracy of the results. ${ }^{20}$ Therefore, it is necessary to investigate the influences of different tetrahedral mesh sizes on the finite element results to select an appropriate mesh size. In this study, the two 3D solid parts were divided into three-dimensional tetrahedral mesh, and the mesh sizes were set to $0.5 \mathrm{~mm}, 0.75 \mathrm{~mm}, 1 \mathrm{~mm}, 1.5 \mathrm{~mm}, 2 \mathrm{~mm}, 2.5 \mathrm{~mm}$ and $3 \mathrm{~mm}$. The composite proximal femur models with three-dimensional tetrahedral mesh and materials were shown in Figure 2a-b. The cortical bone was transparent, and the internal opaque part was cancellous bone. The parameters required in the three numerical methods including Young's modulus, Poisson's ratio, bone density, compressive failure strain, shear modulus and bulk modulus were listed in Table 1.

The distal end of the composite femur was fixed. $\alpha$ angle of $10^{\circ}$ and $\beta$ angle of $15^{\circ}$ were used to simulate the fall posture (As shown in Figere 2c). Loading was exerted on a spherical region with a $35 \mathrm{~mm}$ diameter on the femoral head, and the fracture process was simulated. The maximum principal stress, displacement at point $\mathrm{A}$ in Figure 1a, and the running time of the program were recorded in the models with 7 different mesh sizes mentioned above. Finally, the results of displacement at the point were compared with the results of mechanical tests to obtain the appropriate mesh size.

\subsection{2 | IDE based FEA}

IED based FEA on femur fracture analysis were developed. Through the Python script interacting using Abaqus, the crack propagation was regarded as a new iteration process, and the crack propagation path and fracture load with time history during the impact process were obtained, the detailed workflow is shown in Figure 3. In this study, Young's modulus, bone density, Poisson's ratio, shear modulus, and bulk modulus were assigned to the model according to Table 1 . An initial increment of load was set, and the principal strain of the composite femur was calculated. And the ratio of the tensile failure strain to the compressive failure strain of the composite femur material was set 0.6 according to the literature. ${ }^{15}$ According to the failure strain in Table 1, it was judged whether the element was invalid, and the dynamic load continued to increase with time. During the loading process, principal strain of each element in the composite femur model were compared, when it exceeded the failure strain, it was deleted. The above steps were repeated. When the number of deleted elements reached a certain number, the model would break. 


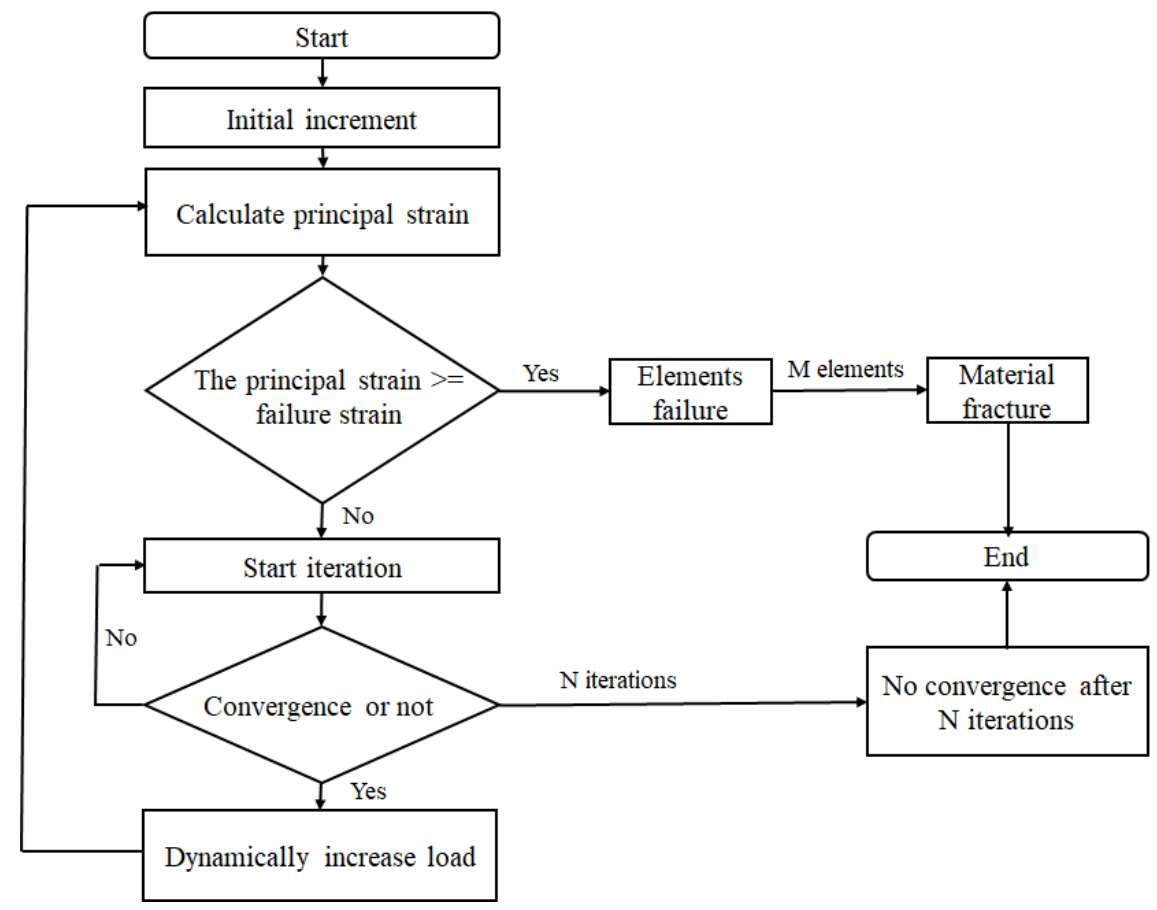

Figure 3 The flowchart of the algorithm.

In this method, the relationship between each node and time in the finite element model is expressed as:

$x_{i}=x_{i}\left(x_{a}, \mathrm{t}\right)(1)$

The initial position at $t_{0}$ is

$x_{i}\left(x_{a}, 0\right)=x_{a}$

$\dot{x}_{i}\left(x_{a}, 0\right)=\sigma_{\mathrm{ij}}\left(x_{a}\right)$

Each node and the whole system satisfy the laws of conservation of momentum, energy and mass:

$\rho \Delta_{\imath} / \Delta \tau=F_{i}+\partial \sigma_{\mathrm{ij}} / \partial x_{j}$

$2 \rho D e / D t=\sigma_{\mathrm{ij}} \bullet\left(\vartheta_{i, j}+\vartheta_{j, i}\right)$

$\rho\left(x_{i}\right)=\rho_{0}\left(X_{a}\right) \bullet\left|[?] \mathrm{X}_{\mathrm{a}} /[?] \mathrm{x}_{\mathrm{i}}\right|$

The boundary conditions of the loading force are as follows:

$\partial b_{1} \sigma_{\mathrm{ij}} \bullet n_{i}=t_{i}(\mathrm{t})(4)$

The displacement boundary conditions are as follows:

$\partial b_{2} x_{i} \bullet\left(x_{a}, t\right)=X_{i}(\mathrm{t})(5)$

The contact inner boundary conditions are as follows:

$\partial b_{3}\left(\sigma_{\mathrm{ij}}^{+}-\sigma_{\mathrm{ij}}^{-}\right) \bullet n_{i}=0\left(x^{+}=x^{-}\right)$

Here, $x_{i}$ was the space position of the i-node, $x_{a}$ was the node coordinates at the initial time, $\dot{x}_{i}$ was the node velocity at the initial time, $\sigma_{\mathrm{ij}}$ was the stress field, $\rho$ was the density, $\vartheta_{i}$ was the component of the velocity on the coordinate $i . F_{i}$ was the unit volume force, $x_{j}$ was the displacement component in the normal direction of the object surface, $e$ was the internal energy t per unit mass, $\vartheta_{j}$ was the unit vector of the velocity on 
the normal line of the outer surface of the object; $\rho\left(x_{i}\right)$ was the density of the object when the node is at the spatial position $x_{i}$, and $\rho_{0}\left(X_{a}\right)$ was the density of the object at the initial moment. $n_{i}$ was the cosine of the outer normal direction on the stress boundary surface. By bringing the above boundary conditions into each node for calculation, the motion law and energy change law of each node can be obtained, that is, the characteristic relationship of the entire femur collision response.

Table 1 Mechanical properties used in the numerical model.

\begin{tabular}{lll}
\hline Property & Cancellous bone & Cortical bone \\
Density $-\rho\left(\mathrm{g} / \mathrm{cm}^{3}\right)^{21}$ & 0.27 & 1.64 \\
Poisson's ratio $-v^{21}$ & 0.3 & 0.3 \\
Young's modulus $-E(\mathrm{MPa})^{21}$ & 155 & 10400 \\
Compressive failure strain $-\epsilon_{\mathrm{S}}{ }^{21}$ & 0.0387 & 0.0165 \\
\hline
\end{tabular}

The XFEM and user subroutine USDFLD were also used to compare with IED based FEA in the femur fracture analysis.

The XFEM method can overcome the shortcoming of large amount of calculation brought by meshing of the crack surface during the simulation of crack propagation path. At the same time, XFEM does not need to preset the crack when simulating the crack propagation path. These advantages make it more widely used in the analysis of fracture mechanical behavior of biological material such as bone. By using the XFEM module in Abaqus/Standard, the virtual crack closure technology (VCCT) was used to simulate the crack propagation process. Pressure loading was applied. The load was continuously increased until the composite femur failed to fracture. The crack propagation path and fracture load of the composite femur were predicted. The Young's modulus, bone density, Poisson's ratio and compressive failure strain required in this method were listed in Table 1 . The critical energy value $G_{C}$ necessary for XFEM to predict the start of crack propagation was estimated from the fracture toughness $K_{c}$, as shown in Eq. (7), and the fracture toughness $K_{c}$ was related to bone density, and can be obtained by Eq. (8). ${ }^{15}$

$G_{C}\left(J M^{-2}\right)=\frac{K_{c}^{2}\left(1-\nu^{2}\right)}{E}(7)$

$K_{c}\left(N m^{-1.5}\right)=0.7413 * \times 10^{6} \times \rho^{1.49} K_{c}\left(N m^{-1.5}\right)=0.7413 \times 10^{6} \times \rho^{1.49}(8)$

where plane strain has been assumed and the ratio between different fracture modes in terms of Gc for human bone was proposed by: ${ }^{22}$

$\mathrm{G}_{\mathrm{IIC}} / \mathrm{G}_{\mathrm{IC}}=\mathrm{G}_{\mathrm{IIIC}} / \mathrm{GIC}=0.33(9)$

Eq. (9) was calculated in $^{22}$ for cortical bone when crack orientation is orthogonal to osteons. In this work we extend this expression for any orientation of the crack. The same relationship has been used in other works for modelling human femur fracture

In subroutine USDFLD, three main material parameters needed to be defined, i.e., Young's modulus, tensile and compressive failure strain of the composite femur material (see Table 1), and the ratio of the tensile failure strain to the compressive failure strain of the composite femur material was set 0.6 according to the literature. ${ }^{15}$ The material degradation method was written in the subroutine. In this study, the parameter degradation method proposed by Giner was used to describe the degradation according to the percentage of Young's modulus ${ }^{23}$ ( the material of no failure is $100 \%, F_{1}=0, F_{2}=0$. the material of compressive failure is $5 \%, F_{1}=1, F_{2}=0$. the material of Tensile failure is $5 \%, F_{1}=0, F_{2}=1$.). In the subroutine, two field variables $F_{1}$ and $F_{2}$ were defined, as shown in Eq. (10) and (11). The Young's modulus of the element was determined according to the values of the field variables $F_{1}$ and $F_{2}$, thereby the crack propagation can be simulated:

$F_{1}=\| /(10)$

$F_{2}=\| /(11)$ 
Here, was the first principal strain, was the third principal strain, was the compressive failure strain, and was the tensile failure strain.

\subsection{The related factors of fracture}

In order to understand the falling risk of the elderly, the numerical model was used to simulate the effects of different impact speeds, fall postures, and cortical thicknesses on fracture types and mechanical responses. In this study, four impact speeds were simulated, the relationships between load and time under the four impact speeds were as follows: $f_{1}=1000 t ; f_{2}=2000 t ; f_{3}=7000 t ; f_{4}=14000 t$. The distal end and the greater trochanter of the composite femur completely were fixed. $a$ angle of $90^{\circ}$ and $\beta$ angle of $30^{\circ}$ were used to simulate the fall posture, and the dynamic pressure loading was applied perpendicular to the direction of the composite femur head. 108 fall postures were described by the two angles defined above (as shown in Figure 2a-b). The corresponding serial numbers were shown in Table 2. The loading conditions set for the 108 models were the same. The distal end and the greater trochanter of the composite femur were completely fixed. The dynamic pressure loading was applied at a speed of $3 \mathrm{~mm} / \mathrm{min}$ on a $35 \mathrm{~mm}$ diameter spherical region above the femoral head. It was performed until the composite femur fractured.

Table 2 Model numbers of 108 different fall postures defined by the combination of angles $a$ and $\beta$.

\begin{tabular}{|c|c|c|c|c|}
\hline$\beta a$ & $0^{\circ}$ & $15^{\circ}$ & $30^{\circ}$ & $45^{\circ}$ \\
\hline $0^{\circ}$ & 1 & 28 & 55 & 82 \\
\hline $5^{\circ}$ & 2 & 29 & 56 & 83 \\
\hline $10^{\circ}$ & 3 & 30 & 57 & 84 \\
\hline $15^{\circ}$ & 4 & 31 & 58 & 85 \\
\hline $20^{\circ}$ & 5 & 32 & 59 & 86 \\
\hline $25^{\circ}$ & 6 & 33 & 60 & 87 \\
\hline $30^{\circ}$ & 7 & 34 & 61 & 88 \\
\hline $35^{\circ}$ & 8 & 35 & 62 & 89 \\
\hline $40^{\circ}$ & 9 & 36 & 63 & 90 \\
\hline $45^{\circ}$ & 10 & 37 & 64 & 91 \\
\hline $50^{\circ}$ & 11 & 38 & 65 & 92 \\
\hline $55^{\circ}$ & 12 & 39 & 66 & 93 \\
\hline $60^{\circ}$ & 13 & 40 & 67 & 94 \\
\hline $70^{\circ}$ & 14 & 41 & 68 & 95 \\
\hline $75^{\circ}$ & 15 & 42 & 69 & 96 \\
\hline $80^{\circ}$ & 16 & 43 & 70 & 97 \\
\hline $85^{\circ}$ & 17 & 44 & 71 & 98 \\
\hline $90^{\circ}$ & 18 & 45 & 72 & 99 \\
\hline $95^{\circ}$ & 19 & 46 & 73 & 100 \\
\hline $100^{\circ}$ & 20 & 47 & 74 & 101 \\
\hline $105^{\circ}$ & 21 & 48 & 75 & 102 \\
\hline $110^{\circ}$ & 22 & 49 & 76 & 103 \\
\hline $115^{\circ}$ & 23 & 50 & 77 & 104 \\
\hline $120^{\circ}$ & 24 & 51 & 78 & 105 \\
\hline $125^{\circ}$ & 25 & 52 & 79 & 106 \\
\hline $130^{\circ}$ & 26 & 53 & 80 & 107 \\
\hline $135^{\circ}$ & 27 & 54 & 81 & 108 \\
\hline
\end{tabular}

It was pointed out that both cortical thickness and cortical thickness index (CTI) were significant risk factors for proximal femoral fracture. ${ }^{24}$ Thus, in order to understand the relative contribution of cortical thickness for the treatment and prevention of fractures in clinics, the composite proximal femur models with 8 different cortical thicknesses were simulated (as shown in Figure 4). Material properties of cancellous 
bone and cortical bone were assigned to elements at different locations. The quality of cortical bone/bone mass was equivalent to CTI, which was set to $100 \%, 80 \%, 60 \%, 40 \%, 20 \%, 10 \%, 0 \%$, to represent different cortical thicknesses. A hollow model was set for comparison. The distal end and the greater trochanter of the composite femur were completely fixed. $a$ angle of $90^{\circ}$ and $\beta$ angle of $30^{\circ}$ were used to simulate the fall posture. The dynamic pressure loading was applied at a speed of $3 \mathrm{~mm} / \mathrm{min}$ on a $35 \mathrm{~mm}$ diameter spherical region above the femoral head. It was performed until the composite femur fractured.
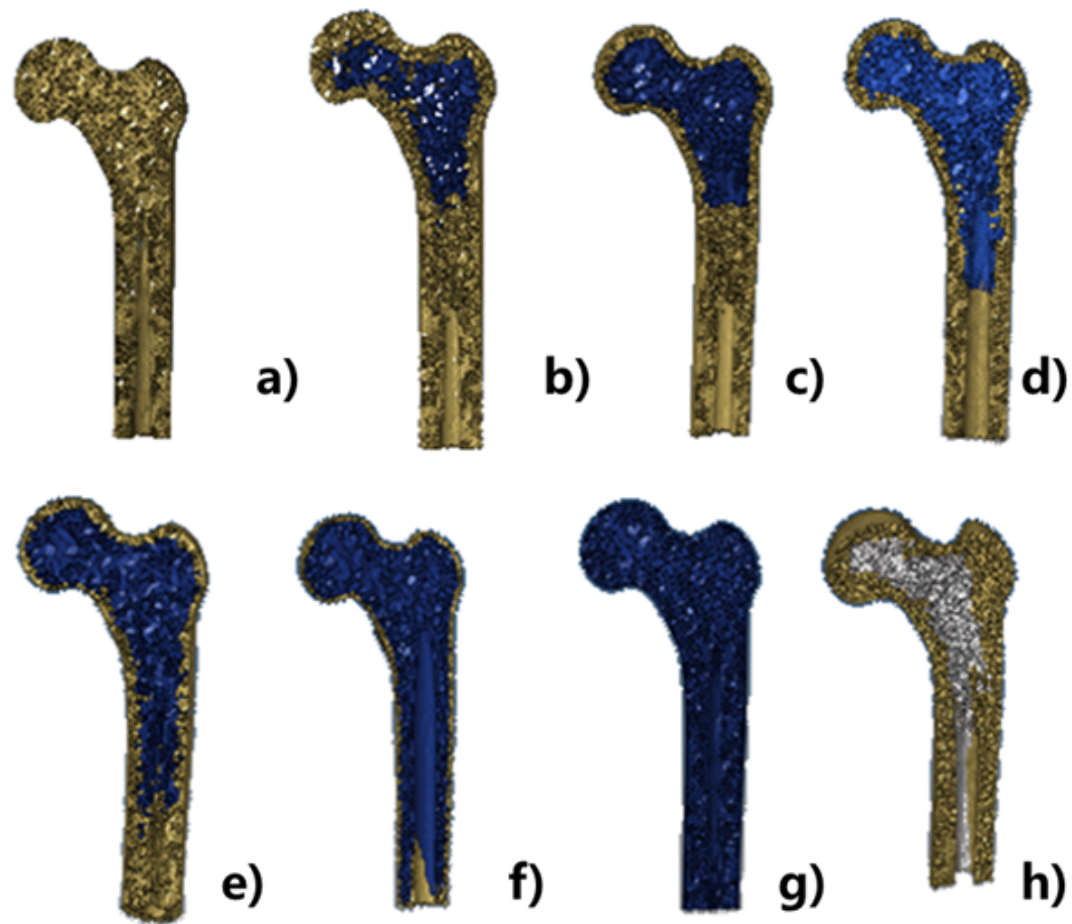

Figure 4 Different thicknesses of cortical bones as defined in the section 2.4, a) 100\%; b) 80\%; c) 60\%; d) $40 \%$; e) $20 \%$; f) $10 \%$; g) $0 \%$; h) Hollow bone model.

\section{3 | Results}

\section{1 | Mesh convergence results}

Figure 5a showed the effect of the ratio of simulated displacement to experimental displacement of position A. It could be seen that the smaller the element size, the higher the displacement accuracy of the finite element analysis. Thus, the element size should be less than or equal to $1.0 \mathrm{~mm}$. It could be seen from Figure $5 \mathrm{~b}$ that in order to reduce the influence of element size on the maximum principal stress of the composite femur and improve the accuracy of finite element analysis, the element size should be less than $2 \mathrm{~mm}$. According to the analysis above, the element size should be selected within the range of $1.0 \mathrm{~mm}$ or less. A good predicted accuracy was found on a fine mesh, while a much longer calculated time was costed (Figure 5c). It was found the running time was 1.5-3 times longer than that of $1 \mathrm{~mm}$ mesh size. Therefore, $1 \mathrm{~mm}$ was selected as the appropriate mesh size in this study. 


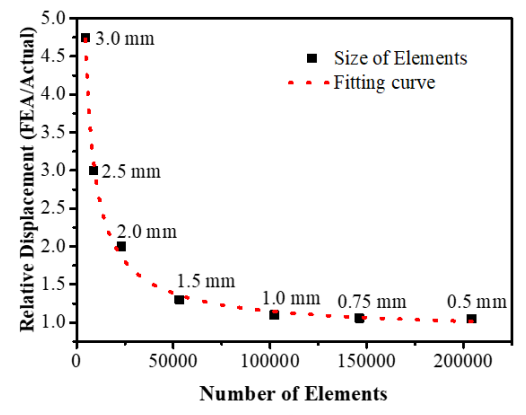

a)

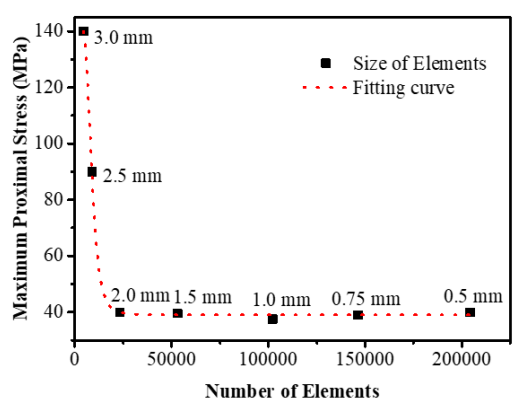

b)

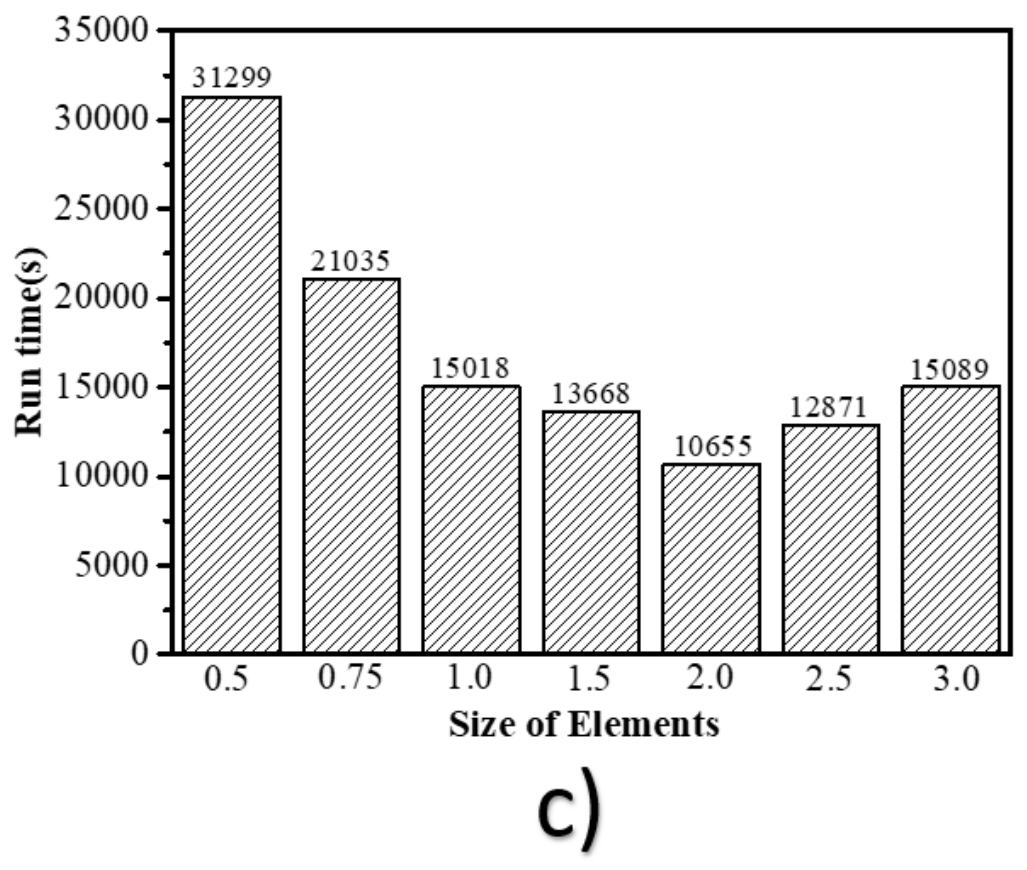

Figure 5 The effects of different mesh sizes on the finite element results, a) The ratio of simulated displacement and experimental displacement of point A. (Point A is shown in Figure 1); b) The maximum principal stress of the composite proximal femur; c) Running time of the finite element analysis.

\section{2 | Comparison of three numerical simulations}

\subsection{1 | Analysis in elastic regime}

The models developed reproduce the mechanical behaviour of the composite femurs and the loading path defined in the experiments. In the elastic regime, femurs were instrumented with 8 strain gages . Since the four measurements were recorded at four different loading steps $(250 \mathrm{~N}, 500 \mathrm{~N}, 7500 \mathrm{~N})$, a total of 24 strain data were registered for each femur to validate the corresponding numerical model. It can be seen that the correlation $\mathrm{R}^{2}$ between the surface strain signal of the artificial composite femur simulated by simulation and the surface strain signal obtained by the composite femur compression experiment is 0.9173 (as shown in Figure 6) 


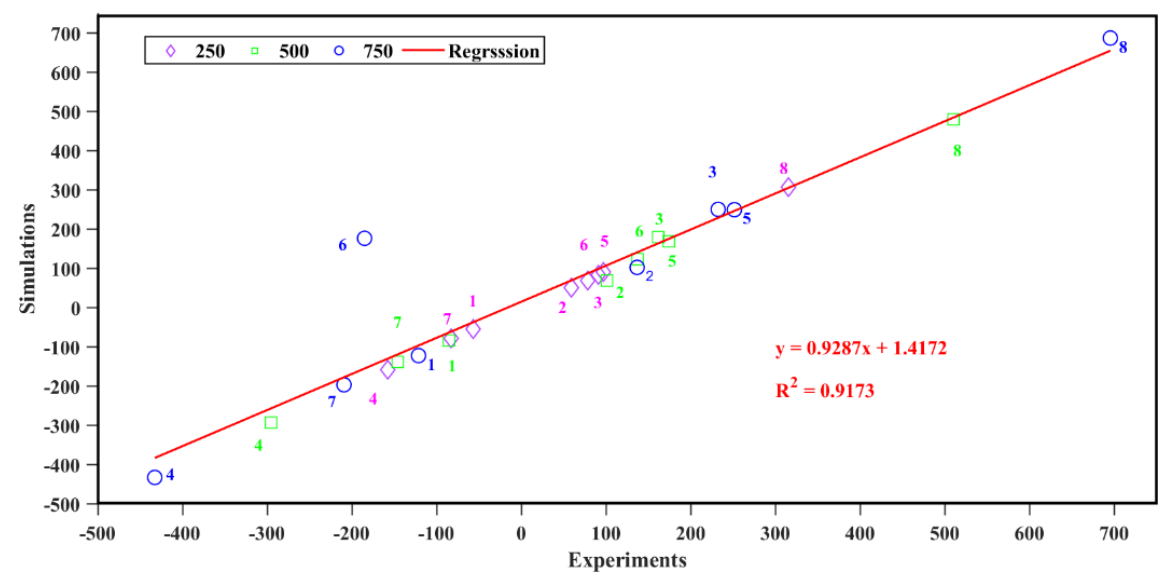

Figure 6 The fitting of the surface strain obtained by simulation and the surface strain obtained by experiment. The numbers in the figure are $250 \mathrm{~N}, 500 \mathrm{~N}$ and $750 \mathrm{~N}$, and different colors represent the strains of different loadings.

\subsection{1 | Fracture load and crack propagation path}

The relative error between the simulated fracture load and the experimental fracture load were listed in Table 3, and the crack propagation paths were shown in Figure 7. In the fracture test of composite femur, the fracture load was $6573 \pm 10 \mathrm{~N}$. It was found that the results of IED based FEA and dynamic simulation of composite femur fractures were closest to the results of mechanical tests. Therefore, in the following study on fracture factors, the IED based FEA was selected for simulation.

Table 3 The relative error between simulated fracture load and the experimental fracture load

\begin{tabular}{llll}
\hline Method & Numerical simulation method & Numerical simulation method & Numerical simulation method \\
& XFEM & USDFLD & IED \\
Numerical model & 5897 & 6057 & 6345 \\
Relative error & $10.2 \%$ & $7.3 \%$ & $3.4 \%$ \\
\hline
\end{tabular}



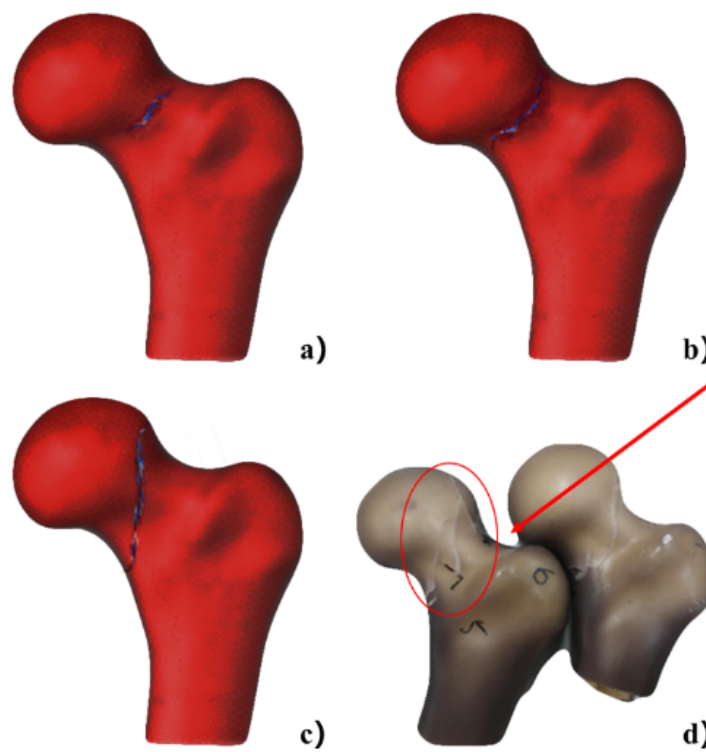

b)

c)

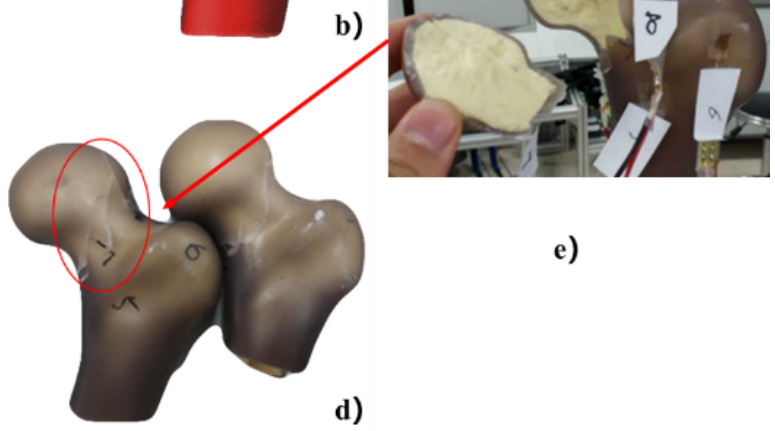

Figure 7 Crack propagation path obtained by simulation and mechanical tests, a) XFEM method; b) User subroutine USDFLD method; c) IED based FEA method; d) crack propagation paths; e) Completely broken.

\section{3 | Simulation results of fracture-related factors}

\subsection{1 | Effects of impact speeds}

Figure 8 showed the effects of impact speeds on the mechanical responses of composite proximal femur fractures. Figure 8a-b showed the fracture load and fracture time decreased with the increase of the impact speed, which meant that the impact speeds were negatively correlated with the fracture loads and fracture time of the composite proximal femurs. Figure $8 \mathrm{c}$ showed the effects of speeds on the crack lengths. It could be seen that when the impact speed was $2000 \mathrm{~mm} / \mathrm{s}$, the crack length of the composite proximal femur fracture was the smallest. When the impact speed was $14000 \mathrm{~mm} / \mathrm{s}$, the crack length of the composite proximal femur fracture was the largest. However, when the impact speed was $1000 \mathrm{~mm} / \mathrm{s}$, the crack length of the composite proximal femur fracture was nearly similar with the crack length when impact speed was $2000 \mathrm{~mm} / \mathrm{s}$. It meant that when impact speed exceeded a certain range, the greater the impact speed, the longer the crack length of the composite femur. Figure $8 \mathrm{~d}$ showed with the increase of impact speed, the instantaneous speed at the time of fracture became greater, and the greater damage to the body. 

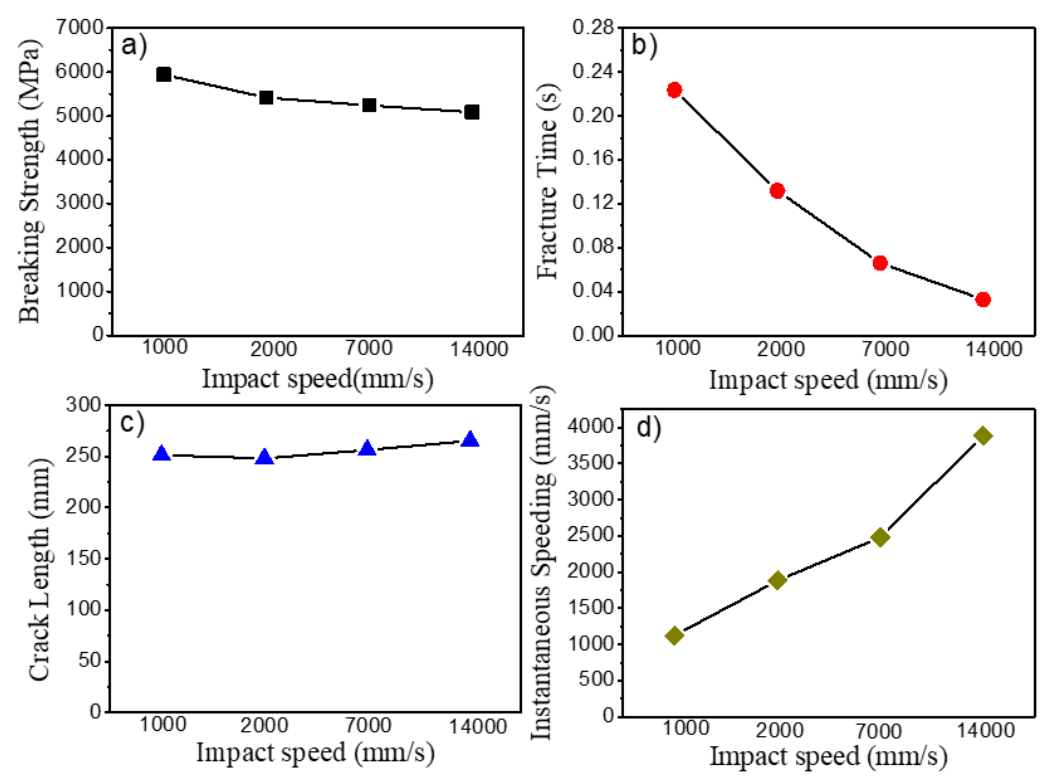

Figure 8 The effects of impact speed on the mechanical responses of composite proximal femoral fractures. a) Breaking strength; b) Fracture time; c) Crack length; d) Instantaneous speed.

Figure 9 showed the simulated crack propagation paths of composite proximal femur under different impact speeds. It could be seen that all the four impact speeds caused femoral neck fractures, and all cracks started from the femoral neck. As the load increased, the crack gradually extended, and finally the entire composite femur fracture occurred. It could also be seen that the crack paths caused by different impact speeds were different. As the impact speed increased, the fracture line gradually thickened from the upper side of the composite femur neck and moved downward to the greater trochanter of the composite femur. 


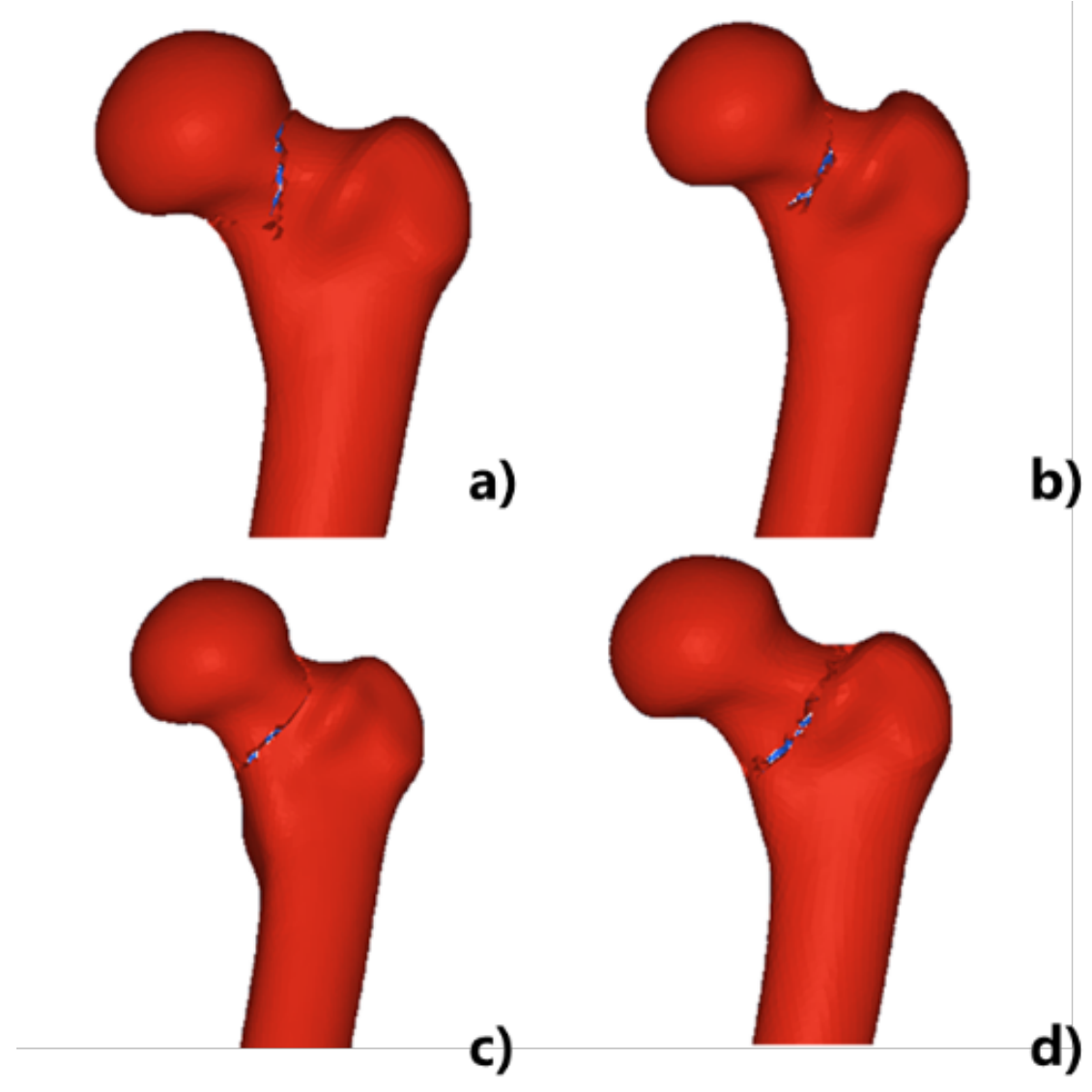

Figure 9 Simulated fracture paths of composite proximal femur with different impact speeds, a) $1000 \mathrm{~mm} / \mathrm{s}$; b) $2000 \mathrm{~mm} / \mathrm{s}$; c) $7000 \mathrm{~mm} / \mathrm{s}$; d) $14000 \mathrm{~mm} / \mathrm{s}$.

\subsection{2 | Effects of fall postures}

When $\beta=0^{\circ}, 15^{\circ}, 30^{\circ}$ and $45^{\circ}$, the relationships between the angle of $a$ and the maximum principal stress, maximum principal strain and fracture time were analyzed. The results were shown in Table 4. It was found that when $\beta=0^{\circ}, 30^{\circ}, 45^{\circ}$, $\alpha$ value had no significant correlation with the maximum principal stress, maximum principal strain and fracture time $(\mathrm{p}>0.05)$. When $\beta=15^{\circ}$, the $\alpha$ angle was significantly correlated with the maximum principal stress of the model $\left(\mathrm{R}^{2}=0.887, \mathrm{p}<0.05\right)$. When $\beta=0^{\circ}, 30^{\circ}, 45^{\circ}$, the $\alpha$ angle was significantly correlated with the maximum principal strain of the model $\left(\mathrm{R}^{2}=0.913, \mathrm{p}<0.05\right)$; however, the $\alpha$ angle had no significant correlation with the model fracture time $\left(\mathrm{R}^{2}=0.689, \mathrm{p}>0.05\right)$ and the maximum principal stress $\left(\mathrm{R}^{2}=0.776, \mathrm{p}>0.05\right)$. When $\beta=15^{\circ}$ and $a=15^{\circ}$, the maximum principal stress and the maximum principal strain of the composite femur model were the smallest. In this case, fracture was the most unlikely to occur, and the fracture load was $7321 \mathrm{~N}$. When $\beta=15^{\circ}$ and $\alpha=135^{\circ}$, the maximum principal stress and the maximum principal strain of the model were the largest. In this case, the fracture was most likely to occur, and the fracture load was $5317 \mathrm{~N}$.

Table 4 When the angle $\beta$ was constant, the p-value between the angle $\alpha$ and the maximum principal stress, the maximum principal strain and fracture time.

\begin{tabular}{llll}
\hline & The maximum principal stress & The maximum principal strain & Fracture time \\
$\beta=0^{\circ}$ & 0.2315 & 0.1259 & 0.2013 \\
$\beta=15^{\circ}$ & $\mathbf{0 . 0 7 5}^{*}$ & $\mathbf{0 . 0 0 8 3}^{*}$ & 0.2312
\end{tabular}



$\beta=30^{\circ} \quad 0.1876$
0.1974

* The data of significant correlation.

When $\beta=15^{\circ}$, as the angle $\alpha$ increased, the maximum principal strain of the composite femur decreased, the element failure occurred easier, the more likely the fracture is to occur. Through iterative calculations, the composite femur model would break as the failed elements accumulated to a certain degree. Thus, the smaller the maximum principal strain, the less prone to fracture. In other words, falling forward was less likely to cause composite proximal femur fracture than falling backward. Eugenio et al. ${ }^{25}$ found that for falling backward, if one could rotate the body forward or sideways, the risk of proximal femoral fracture could be reduced, which was consistent with the numerical simulation results of this study. In addition, due to the presence of vascular perforation holes on the outer and upper side of the femoral neck, the bone cortex was thinner, and the bone mass loss rate was faster than the inner and lower sides. ${ }^{26}$ When a fall occurred, the load on the outer and upper sides of the femoral neck were compressed, while the femoral neck was under tension at the inner and lower side. Thus, a shear force was formed at the femoral neck in instantaneous time, which made the composite proximal femur more likely to cause femoral neck fracture. Figure 10 showed eight representative fracture crack propagation paths under different fall postures. It could be seen that different fall postures would lead to two different fracture types: composite femur neck fracture and intertrochanteric fracture.
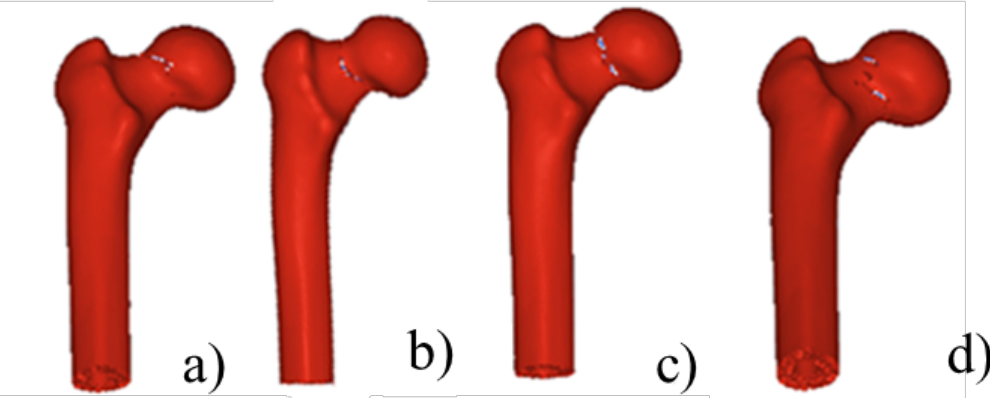

d)
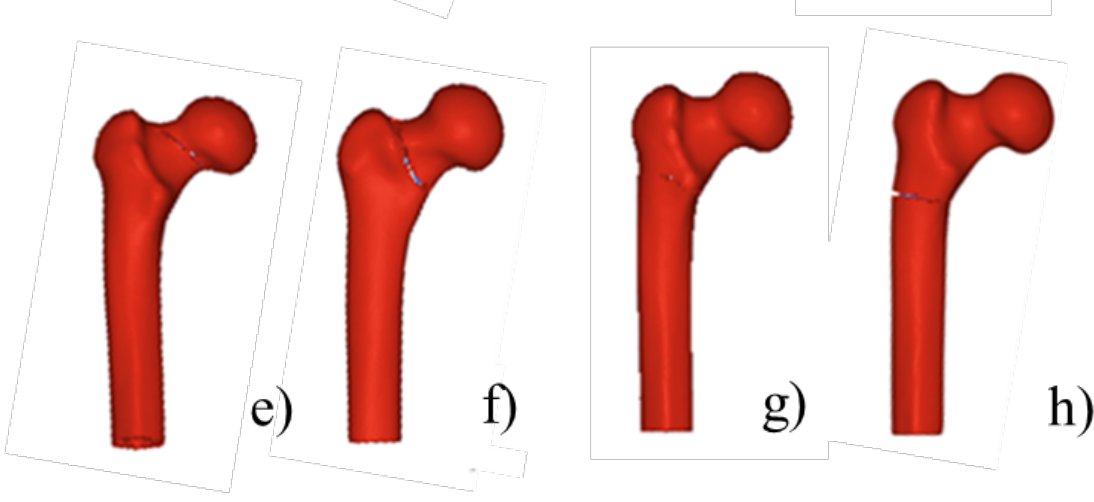

Figure 10 Eight typical fracture paths obtained by numerical simulation, including femoral neck fracture and intertrochanteric fracture, the numbers correspond to the different loading conditions listed in Table 2, a) $\alpha=20^{\circ}, \beta=0^{\circ}$; b) $\alpha=75^{\circ}, \beta=0^{\circ}$; c) $\alpha=0^{\circ}, \beta=15^{\circ}$; d) $\alpha=55^{\circ}, \beta=0^{\circ}$; e) $\alpha=0^{\circ}, \beta=30^{\circ}$; f) $\alpha=40^{\circ}, \beta=30^{\circ}$; g) $\alpha=100^{\circ}, \beta=30^{\circ}$; h) $\alpha=80^{\circ}, \beta=45^{\circ}$.

\subsubsection{Effects of cortical thicknesses}


The mechanical responses of the models with 8 different cortical thicknesses were listed in Table 5 . It could be seen that the maximum principal stress and the maximum principal strain of the composite femur model with $100 \%$ cortical bone mass/integral bone mass were $140.6 \mathrm{MPa}$ and $0.994 \times 10^{-2}$, respectively, and the fracture load was $7329 \mathrm{~N}$. The maximum principal stress and the maximum principal strain of the composite femur model with the cortical bone mass/integral mass of $0 \%$ were the largest in the eight models, which were $397.9 \mathrm{MPa}$ and $1.301 \times 10^{-2}$, respectively, and the fracture load was $4575 \mathrm{~N}$. It showed that the thicker the proximal cortical bone of the composite femur, the smaller the maximum principal strain of the model is.

Table 5 Effects of different cortical thicknesses on the mechanical responses.

\begin{tabular}{|c|c|c|c|c|c|c|c|}
\hline \multirow[t]{2}{*}{$\begin{array}{l}\text { Cortical } \\
\text { bone qual- } \\
\text { ity/Osteopathic } \\
\text { quality }\end{array}$} & $\begin{array}{l}\text { Cortical } \\
\text { bone } \\
\text { c }\end{array}$ & $\begin{array}{l}\text { Cortical } \\
\text { bone }\end{array}$ & $\begin{array}{l}\text { Cancellous } \\
\text { bone }\end{array}$ & $\begin{array}{l}\text { Cancellous } \\
\text { bone }\end{array}$ & $\begin{array}{l}\text { Fracture } \\
\text { load }(\mathrm{N})\end{array}$ & $\begin{array}{l}\text { Maximum } \\
\text { principal } \\
\text { stress }(\mathrm{Pa})\end{array}$ & $\begin{array}{l}\text { Maximum prir } \\
\text { cipal } \\
\text { strain }\end{array}$ \\
\hline & Elements & Nodes & Elements & Nodes & & & \\
\hline $100 \%$ & 80693 & 14748 & - & - & 7329 & $1.406 \times 10^{8}$ & $0.994 \times 10^{-2}$ \\
\hline $80 \%$ & 77649 & 13742 & 3044 & 1006 & 7100 & $2.945 \times 10^{8}$ & $1.209 \times 10^{-2}$ \\
\hline $60 \%$ & 68650 & 11742 & 12043 & 3016 & 6550 & $3.197 \times 10^{8}$ & $1.234 \times 10^{-2}$ \\
\hline $40 \%$ & 54425 & 9391 & 26268 & 5391 & 6345 & $3.557 \times 10^{8}$ & $1.276 \times 10^{-2}$ \\
\hline $20 \%$ & 48918 & 8773 & 31775 & 5945 & 5701 & $3.979 \times 10^{8}$ & $1.278 \times 10^{-2}$ \\
\hline $10 \%$ & 33918 & 5773 & 46775 & 8975 & 5039 & $3.979 \times 10^{8}$ & $1.291 \times 10^{-2}$ \\
\hline $0 \%$ & - & - & 70693 & 14748 & 4575 & $3.979 \times 10^{8}$ & $1.301 \times 10^{-2}$ \\
\hline $\begin{array}{l}100 \% \\
\text { (Hollow) }\end{array}$ & 54425 & 9391 & - & - & 6243 & $3.457 \times 10^{8}$ & $1.076 \times 10^{-2}$ \\
\hline
\end{tabular}

Figure 11 was the crack propagation paths of the cortical bone models with different thicknesses. It could be seen that when the model contained cortical bone, a crack began to initiate from the femoral neck, and it gradually extended upward.
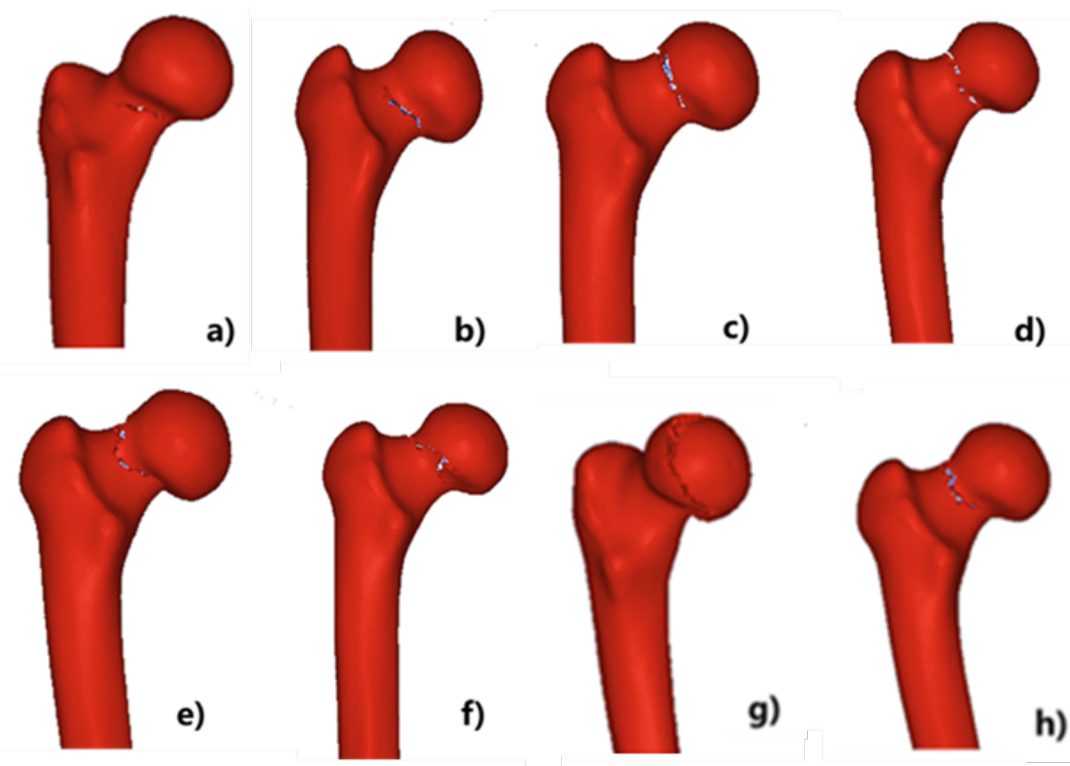

Figure 11 Crack propagation paths of the composite femur models with different thicknesses of cortical 
bones as defined in the section 2.4 , a) $100 \%$; b) $80 \%$; c) $60 \%$; d) $40 \%$; e) $20 \%$; f) $10 \%$; g) $0 \%$; h) Hollow bone model.

\section{4 | Discussions}

Numerical simulation has been considered as an efficient approach to predict the risk of proximal femur fracture or the types of fractures after failing. Besides, it is important to clarify the effects of different fall postures, impact speeds and cortical thicknesses on the fracture types and mechanical responses for indicating the femoral fracture risk in the elderly. In this study, IED based FEA was developed to simulate the fracture behaviors of composite proximal femurs with a comparison of XFEM and USDFLD, and the results of numerical simulations were verified by mechanical tests. In addition, the effects of different fall conditions (fall postures, impact speeds) and femoral cortical thicknesses on fracture types and mechanical responses were comprehensively investigated.

A better prediction accuracy on the occurrence and development of composite proximal femur fracture was found on the IED based FEA than XFEM and USDFLD. In Figure 7, it could be observed that the fracture path was very similar for all methods since all the three methods approach used in this study modeled the onset of cracking using the maximum principal strain yield criterion. The predicted fracture paths were in good agreement with experimental results, while the IED based FEA method leads to longer fracture paths than the XFEM and USDFLD. In addition, the results of IED based FEA were closest to the results of mechanical tests. This method presented good behaviour concerning convergence through incremental crack growth, thus leading to more realistic results.

The results of this study showed that the fracture types, fracture loads, and crack propagation paths of composite proximal femurs were significantly affected by the fall postures, impact speeds, and cortical thicknesses. As the impact speed increased, the complexity and roughness of fracture cracks also increased, which were consistent with the results in the literatures. ${ }^{27,28}$ When the fall postures were different, it was found that with the increase of $a$ angle, the maximum principal stress and the maximum principal strain of the femur also increased. Falling forward was less likely to cause composite proximal femoral fracture compared with falling backward. The fracture load decreased by $27.37 \%$ as $\alpha$ angle changed from $15^{\circ}$ to $135^{\circ}$. Ford and Kryak et al. ${ }^{29,30}$ also reached a conclusion that was basically consistent with the current study, which pointed out that a $26 \%$ reduction in load capacity was equivalent to the result of bone mineral density loss as 25 years after the age of 65 . It could be seen that the cortical thicknesses had great effects on the fracture types and mechanical responses. As the cortex became thinner, the fracture line gradually moved from the composite femoral neck to the intertrochanter, resulted in different types of composite proximal femoral fractures. It could be seen that the thickness of cortical bone was an important factor affecting the types of composite proximal femur fractures. Henning et al. ${ }^{31}$ have reached similar conclusions. The study also pointed out that the thinning of cortical bone with aging led to a gradual decrease in the strength and stiffness of proximal femur, which was the main factor that caused the elderly to be more prone to fracture. ${ }^{32}$ Cortical bone is the main load-bearing region of bone. In the femoral neck, cortical bone contributes higher than cancellous bone to bone strength by $4.6 \%-17.3 \%,{ }^{33}$ but cancellous bone plays an important role in resisting bending deformation. Cortical and cancellous bones are in different locations and have different relative contributions to bone strength. It was pointed out that both cortical thickness and cortical thickness index (CTI) were significant risk factors for proximal femoral fracture. ${ }^{34,35}$ Thus, it was essential to understand the relative contribution of cortical thickness for the treatment and prevention of fractures in clinics.

The change of fall speeds and postures will cause great energy absorption, and different cortical thicknesses in the proximal femur will cause different structural changes, which may be one of the main factors leading to the proximal femur fracture. ${ }^{36}$ Because the XFEM and user subroutine USDFLD cannot simulate sudden changes in the proximal femoral speed and acceleration during the falling process, so the results were quite different from the results of mechanical tests. In this study, IED based FEA could be effective to simulate different fall conditions, and the crack propagation path and fracture load with time during the fall were analyzed. The model could also be used to predict proximal femur fractures caused by other conditions such as dropping from high places or impact in various situations. Also, it could help to understand the 
biomechanical mechanism of proximal femur fracture by investigating the crack propagation path and the final damage state. The model could also be assembled with internal fixation devices to simulate proximal femur internal fixation and simulation verification of mechanical properties of existing metal implants to guide the improvement of internal fixation devices and intraoperative fixation decisions. ${ }^{37,38}$

However, this study still had some limitations and needed to be further improved. Only the composite femur was used to establish a numerical model, in which simply two different material properties of cortical bone and cancellous bone were assigned. Although Graba et al. ${ }^{39}$ showed that composite femurs can replace human bones to do mechanical tests, the composite femurs cannot completely simulate human femurs, since the materials and morphology of human femurs are more complex. In the future, the material properties which are closer to the real human femur should be used. At the same time, only the femur was modeled in this study, and the muscles, fascias and ligaments were not simulated. In the future, mechanical tests could be performed to obtain the loads of muscles, fascias and ligaments on bones, ${ }^{40}$ and a new finite element model of proximal femur would be established. The results obtained would be closer to the reality. Although this study did not carry out direct experimental verification of all models, the crack propagation path and the predicted fracture load in the simulated standing state are highly similar to those obtained by mechanical tests. Thus, the computational model could be considered effective and be used to analyze other conditions. It could be further used to simulate bone implants, fractures caused by pedestrian impacts with cars, and more types of fractures. Besides, it could provide a more theoretical basis for fracture research.

\section{5 | Conclusions}

In this study, an IED based FEA method was developed to simulate fracture of composite femur compared to XFEM and USDFLD for an understanding of the mechanism of proximal femoral fracture. The results of three numerical methods were verified by mechanical tests. By comparing the fracture loads, surface strains and crack propagation paths obtained by numerical simulation and mechanical tests, it was found that the results obtained from the IED based FEA were better than the other two methods, which verified the superiority of this method.

IED based FEA was further used to simulate the effects of different impact speeds, fall postures, and cortical thicknesses on fracture types and mechanical responses. It was found that the changes in impact speeds caused different crack propagation behavior of femoral necks. If the speed became faster, the time of fracture became shorter. Compared with the crack at a lower speed, the crack line caused at a higher speed would move down significantly, affecting the type of proximal femoral fracture. As the fall posture changed, the mechanical response of the composite femur also changed. Falling forward was less likely to cause proximal femoral fracture compared with falling backward. It was also found the model with thin cortical bone was prone to fracture. when the thickness of cortical bone became thinner, the maximum principal strain at break was larger, and the fracture of proximal femur was easier.

This study helped a deep understanding of the fracture process and provided a theoretical basis and method for improving the accuracy of predicting femoral fracture risk.

\section{Acknowledgements}

This work was supported by the National Natural Science Foundation of China (grant number 51705336), Japan Society for the Promotion of Science KAKENHI Grant (grant number 20K20162), Shenzhen Basic Research Project (grant number JCYJ20190807144001746), Shenzhen Industry and Information Technology Foundation (grant number 20180309163834680).

\section{Author Contribution Statement}

Yangyang Cui: Writing-original draft, preparation, Formal analysis Investigation,

Methodology, Data curation.

Dingding Xiang: Project administration, Conceptualization, Methodology. 
Liming Shu: Investigation, Data curation, Writing - review \& editing.

Zhenhua Liao: Validation, Resources.

Zhilin Zhang: Writing - review \& editing.

Weiqiang Liu: Supervision.

\section{Nomenclature}

\begin{tabular}{ll}
\hline XFEM & Extend finite element method \\
\hline USDFLD & User subroutine to redefine field variables \\
IED & Incremental element deletion \\
$a$ & Long axis of the femur in the frontal plane \\
$\beta$ & Femoral neck axis in the horizontal plane \\
$i$ & The node number \\
$j$ & The outer normal direction \\
$x_{i}$ & Space position of the i-node \\
$x_{a}$ & Node coordinates at the initial time \\
$\dot{x}_{i}$ & Node velocity at the initial time \\
$\sigma_{\mathrm{ij}}$ & Stress field \\
$\rho$ & Density \\
$\vartheta_{i}$ & Component of the velocity at the coordinate i \\
$F_{i}$ & Unit volume force \\
$x_{j}$ & Displacement component in the normal direction of surface \\
$e$ & Internal energy per unit mass \\
$\vartheta_{j}$ & Unit vector of the velocity in the normal direction of surface \\
$\rho\left(x_{i}\right)$ & Density at the spatial position $x_{i}$, \\
$\rho_{0}\left(X_{a}\right)$ & Density at the initial moment \\
$n_{i}$ & Cosine of the outer normal direction on the stress boundary surface \\
$\varepsilon_{1}$ & The first principal strain \\
$\varepsilon_{3}$ & The third principal strain \\
$\varepsilon_{c}$ & The compressive failure strain \\
$\varepsilon_{t}$ & The tensile failure strain \\
$v$ & Poisson's ratio \\
$E$ & Young's modulus \\
$F$ & Field variable \\
$f$ & Load \\
$t$ & Time \\
\hline & \\
\hline
\end{tabular}

\section{References}

1. Ettinger MP. Aging bone and osteoporosis: strategies for preventing fractures in the elderly. Archives of Internal Medicine. 2003;163(18):2237-2246.

2. Franz M, Michael G, Christina R, Bernd F. Infection versus hematoma following surgical treatment of proximal femoral fractures in geriatric patients. Geriatric Orthopaedic Surgery \& Rehabilitation. 2018;9(9):215-221.

3. Cooper C, Cole ZA, Holroyd CR, Earl SC, Harvey NC, Dennison EM, et al.. The IOF CSA working group on fracture epidemiology: secular trends in the incidence of hip and other osteoporotic fractures. Osteoporosis International. 2011;22(5):1277-1288.

4. Fyhrie DP; Vashishth D. Bone stiffness predicts strength similarly for human vertebral cancellous bone in compression and for cortical bone in tension. Bone. 2000;26(2):169-173. 
5. Liu FC, Halsey JN, Oleck NC, Lee ES, Granick MS. Facial fractures as a result of falls in the elderly: concomitant injuries and management strategies. Craniomaxillofacial Trauma \& Reconstruction. 2019;12(1):045-053.

6. Supanc V, Sonicki Z, Vukasovic I, Solter VV, Zavoreo I, Kes VB. The role of classic risk factors and prothrombotic factor gene mutations in ischemic stroke risk development in young and middle-aged individuals. Journal of Stroke and Cerebrovascular Diseases. 2016;23(3):171-176.

7. Nakamura T, Takata T. Risk factors related to type of proximal femoral fractures. Orthopedics \& Traumatology. 2010; 47(3):835-837.

8. Cristofolini L, Schileo E, Juszczyk M, Taddei F, Martelli S, Viceconti M. Mechanical testing of bones: the positive synergy of finite-element models and in vitro experiments. Philosophical Transactions of the Royal Society A: Mathematical Physical and Engineering Sciences. 2010;368(1920):2725-2763.

9. Li SM, Abdel-Wahab A, Silberschmidt VV. Analysis of fracture processes in cortical bone tissue. Engineering Fracture Mechanics. 2013;110 (3): 448-458.

10. Nicolas N, Dolbow J, Belytschko T. A finite element method for crack growth without remeshing. International Journal for Numerical Methods in Engineering. 2015; 46(1):131-150.

11. Giambini H, Qin XL, Dragomir-Daescu D, An KN, Nassr A. Specimen-specific vertebral fracture modeling: a feasibility study using the extended finite element method. Medical \& Biological Engineering \& Computing. 2016; 54(4):583-593.

12. Grasa J, Bea JA, Doblare M. A Probabilistic Extended Finite Element Approach: Application to the Prediction of Bone Crack Propagation. Key Engineering Materials. 2007;348-349:77-80.

13. Shu LM, Sugita N. Analysis of fracture, force, and temperature in orthogonal elliptical vibrationassisted bone cutting. Journal of the Mechanical Behavior of Biomedical Materials. 2020;103:599-599.

14. Giner E, Arango C, Vercher A, Fuenmayor FJ. Numerical modelling of the mechanical behaviour of an osteon with microcracks. Journal of the Mechanical Behavior of Biomedical Materials.2014;37:109-124.

15. Ali AA, Cristofolini L, Schileo E, Hu HX, Taddei F, Kim RH, et al.. Specimen-specific modeling of hip fracture pattern and repair. Journal of Biomechanics. 2014;47(2):536-543.

16. Basso T, Klaksvik J, Foss OA. Statistical consequences of using bone mineral density to pair cadaver femurs in comparative ex vivo hip fracture studies. Bone \& Joint Research. 2014;3(11):317-320.

17. Haentjens P, Magaziner J, Colon-Emeric CS, Vanderschueren D, Milisen K, Boonen S. Meta-analysis: excess mortality after hip fracture among older women and men. Annals of Internal Medicine. 2010;152(6):380-387.

18. Weil YA, Safran O, Greenberg A, Mosheiff R, Liebergall M, Khoury A. Outcome of proximal femoral fractures caused by cycling in the young and mid-aged. Injury. 2014; 45(8):1251-1255.

19. Watts NB. Fundamentals and pitfalls of bone densitometry using dual-energy X-ray absorptiometry (DXA). Osteoporosis International. 2004;15(11): 847-854.

20. Baraldi D, Boscato, Brito D. Discrete and finite element models for the analysis of unreinforced and partially reinforced masonry arches. Key Engineering Materials. 2019; 817(1):229-235.

21. Marco M, Giner E, Larrainzar-Garijo R, Caeiro JR, Miguelez MH. Modelling of femur fracture using finite element procedures. Engineering Fracture Mechanics. 2018;15(4):1-11.

22. Zimmermann EA, Launey ME, Barth HD, Ritchie RO. Mixed-mode fracture of human cortical bone. Biomaterials. 2009;30(29):5877-5884.

23. Doitrand A, Leguillon D. Comparison between 2D and 3D applications of the coupled criterion to crack initiation prediction in scarf adhesive joints. International Journal of Adhesion \& Adhesives. 2018;85(2):69-76.

24. Pellegrini A, Tacci F, Leigheb M, Costantino C, Pedrazzini A, Pedrazzi G, et al.. Injuries of the trochanteric region: can analysis of radiographic indices help in prediction of recurrent osteoporotic hip fractures. Acta Bio Medica Atenei Parmensis. 2017;88(4):43-49.

25. Giner E, Arango C, Vercher A, Fuenmayor FJ. Numerical modelling of the mechanical behaviour of an osteon with microcracks. Journal of the Mechanical Behavior of Biomedical Materials. 2014;37(5):109124.

26. Wang ZG, Li JB, Gao F, Weber WJ. Tensile and compressive mechanical behavior of twinned silicon 
carbide nanowires. Acta Materialia. 2010;58(6) :1963-1971.

27. Zhuang H, Li YZ, Lin JK, Cai DL, Cai SQ, Yan LS, et al.. Cortical thickness in the intertrochanteric region may be relevant to hip fracture type. Bmc Musculoskeletal Disorders. 2017;18(1):305-309.

28. Pellegrini A, Tacci F, Leigheb M, Costantino C, Pedrazzini A, Pedrazzi G, et al.. Injuries of the trochanteric region: can analysis of radiographic indices help in prediction of recurrent osteoporotic hip fractures? Acta Bio Medica Atenei Parmensis. 2017;88(4S):43-49.

29. Fleps I, Vuille M, Melnyk A, Ferguson SJ, Guy P, Helgason B, et al.. A novel sideways fall simulator to study hip fractures ex vivo. Plos One. 2018;13(7):201-215.

30. Ford CM, Keaveny TM, Hayes WC. The effect of impact direction on the structural capacity of the proximal femur during falls. Journal of Bone \& Mineral Research. 1996;11(3):377-383.

31. Wu JY, Henning P, Sjogren K, Koskela A, Tuukkanen J, Moverare-Skrtic S, et al.. The androgen receptor is required for maintenance of bone mass in adult male mice. Molecular and Cellular Endocrinology. 2019;479(2):159-169.

32. Gao JZ, Gong H, Zhang R, Zhu D. Age-related regional deterioration patterns and changes in nanoscale characterizations of trabeculae in the femoral head. Exp Gerontol. 2015;62(1):63-72.

33. Zeli Z, Chao WU, Xu L, Haigang H. Effectiveness comparison between minimally invasive surgery and traditional open reduction internal fixation in treatment of unstable distal radial fractures. Chinese Journal of Reparative \& Reconstructive Surgery. 2018;32(3):322-328.

34. Guerrero-Miguel DJ, Alvarez-Fernandez MI, Garcia-Fernandez CC, Gonzalez-Nicieza C, MenendezFernandez C. Analytical and numerical stress field solutions in the Brazilian Test subjected to radial load distributions and their stress effects at the centre of the disk. Journal of Engineering Mathematics. 2019;116(1):29-48.

35. Kassapoglou C, Bauer G. Composite plates under concentrated load on one edge and uniform load on the opposite edge. Mechanics of Advanced Materials \& Structures. 2010;17(3):196-203.

36. Bogdanova O. Studying of fracture of the orthotropic elastic and visco-elastic plates with periodic system of collinear cracks. International Conference on Theoretical, Springer, Cham. 2018; 5:235-236.

37. Xiang DD, Wang P, Tan XP, Chandra S, Wang C, Nai ML, et al.. Anisotropic microstructure and mechanical properties of additively manufactured Co-Cr-Mo alloy using selective electron beam melting for orthopedic implants. Materials Science and Engineering A. 2019;765. https://doi.org/10.1016/j.msea.2019.138270

38. Xiang DD, Sui XD, Tan XP, Hao JY, Wang ZW, Liao ZH, et al.. Improving biotribological properties and corrosion resistance of CoCrMo alloy via a Cr-GLC nanocomposite film in simulated body fluids. Surface and Coatings Technology. 2019;378. https://doi.org/10.1016/j.surfcoat.2019.07.064

39. Graba M. Experimental and numerical analysis of fracture in $41 \mathrm{Cr} 4$ steel-issues of the stationary cracks. International Journal of Applied Mechanics \& Engineering. 2018;23(1):37-63.

40. Shu LM, Yamamoto K, Yao J , Saraswat P, Liu Y, Mitsuishi M, et al.. A subject-specific finite element musculoskeletal framework for mechanics analysis of a total knee replacement. Journal of Biomechanics. 2018;77:146-154. 